\title{
Analysis on the Tendon Spacing of Prestressed Tunnel Liner
}

\author{
Rui JING, Jingfu KANG, Zhen WANG, Zhaowei SHEN, Dazhi WANG
}

\begin{abstract}
In this paper, based on cylindrical shell theory of elastic mechanics, the mechanical property of prestressed concrete lining structure was studied; models of calculating infinitely-long structure and semi-infinitely-long structure were established; theoretical formulas of normal displacement and inner force on the middle surface were deduced; the maximum influence scope of stress was determined and the superposition formula of maximum cable spacing was deduced. Combing observed data in practical project, the theoretical result and measured result of circumferential stress in prestressed concrete lining structure was compared. Results reveal that the theoretical result coincides with the measured value and that the method determining maximum cable spacing can be applied into practical projects. The even distribution of circumferential stress in the axial direction caused by the tensile force of anchor cable is not a factor determining the cable spacing.
\end{abstract}

Keywords: elastic theory; measured data; prestressed concrete lining structure; tendon spacing

\section{INTRODUCTION}

In recent years, prestressed concrete lining structure has been applied into many hydraulic tunnels where the internal water pressure is higher while the geological conditions of wall rock are poor, such as Tunnel passing through the Yellow River and Dahuofang Reservoir in Liaoning. Structural features of prestressed concrete lining are: the prestress long anchor cable in ring directions is evenly distributed at certain intervals on the concrete lining, which makes it possible to produce compressive prestress on the lining against the internal water pressure from the stretch-draw of anchor cable [1]. There are two factors mainly influencing the spacing: one is the strength of prestress and the other one is the even distribution in axial direction of ring prestress.

At present, it has been stipulated in Hydraulic Tunnel Design Specification DL/T5195-2004 [2] that the spacing should be determined by calculating, but it should be within $0.5 \mathrm{~m}$. However, the principle of calculating and the reason why it should be within 0.5 have not been proposed. Jingfu Kang et al [3] investigate the performance of the post-prestressed tunnel liner by fullscale model tests and field instrument of the Xiao Langdi sediment which is the first water convey tunnel constructed using the post-prestressed method in the world. Computational modelling of these situations is becoming increasingly prominent and ranges, for example, from analysis of bolt lengths [4] to parametric studies of the behaviour of simple liner-rock models [5]. Gabriel presents a framework to identify and evaluate the variables that control tunnel behaviour and thus determine the key decisions made in the design of a pressure tunnel [6]. J. L. G. and J. C. [7] describe the parametric studies for assessing the stability of stiffened steel liners in hydroelectric pressure tunnels. Yin Juan et al [8] have analysed through finite element software the influence of different spacings on the strength and stability of lining structure. However, during the structural design of prestressed tunnel liner, the calculated method of the anchor tendon spacing has not been put forward.

For this reason, based on cylindrical shell theory of elastic mechanics and engineering projects, the mechanical property of prestressed concrete lining structure and the theoretic method of setting largest spacing of anchor cable were studied in this paper.

\section{THEORETICAL ANALYSIS \\ 2.1 Basic Assumptions}

According to Theory of Elastic Mechanics [9], the basic assumptions are as follows:

1) The liner concrete was seen as an isotropous elastomer during the study on mechanical property;

2) Liner concrete conforms to plane cross-section assumption before and after the deformation; and surface curvature of inside and outside surfaces of liner concrete on the same cross section is the same;

3) After the stress, displacement and deformation of liner concrete are too small.

\subsection{Theoretical Model of Elastic Mechanics}

The prestressed concrete lining structure under the force of prestressed anchor cable is a cylindrical shell bearing the force distributed in axial symmetry. With single anchor cable, the deformation and internal force produced on the cylinder wall have a range of influence along the axis of cylinder which is called influence range of stress. A model of elastic mechanics was established to study the influence range of stress when the tensile force of single anchor cable was certain.

The length of this cylindrical structure is $L$, the diameter of middle surface is $R$ and the thickness of wall is $\delta$, all of which are shown as follows:

Circumferential force will be applied to the concrete liner once the prestressed anchor cable is pulled. To simplify the calculation, this circumferential force is seen as the equivalent to an evenly-distributed radial pressure $F_{\mathrm{P}}$ along the per unit length and the direction pointing to the center of cylinder cross section is positive (Fig. 1a). Width of location $x$ far away from the cross section while prestressed anchor cable is placed is $d x$, the micro-fission was the object of study (Fig. 1b). While the cylindric structure is under the force of $F_{\mathrm{P}}$, an axial force $N_{\alpha}$ will be produced on the cross section where the micro-fission is perpendicular to $\alpha$-axis and circumferential force $N_{\beta}$ and circumferential moment $M_{\beta}$ will emerge on the cross section where the fission is perpendicular to $\beta$-axis. $N_{\alpha}$ 
and $N_{\beta}$ subjected to tension are positive; $M_{\alpha}$ and $M_{\beta}$ tensing outside of the cylinder are positive, pointing to the outside are positive.

a)
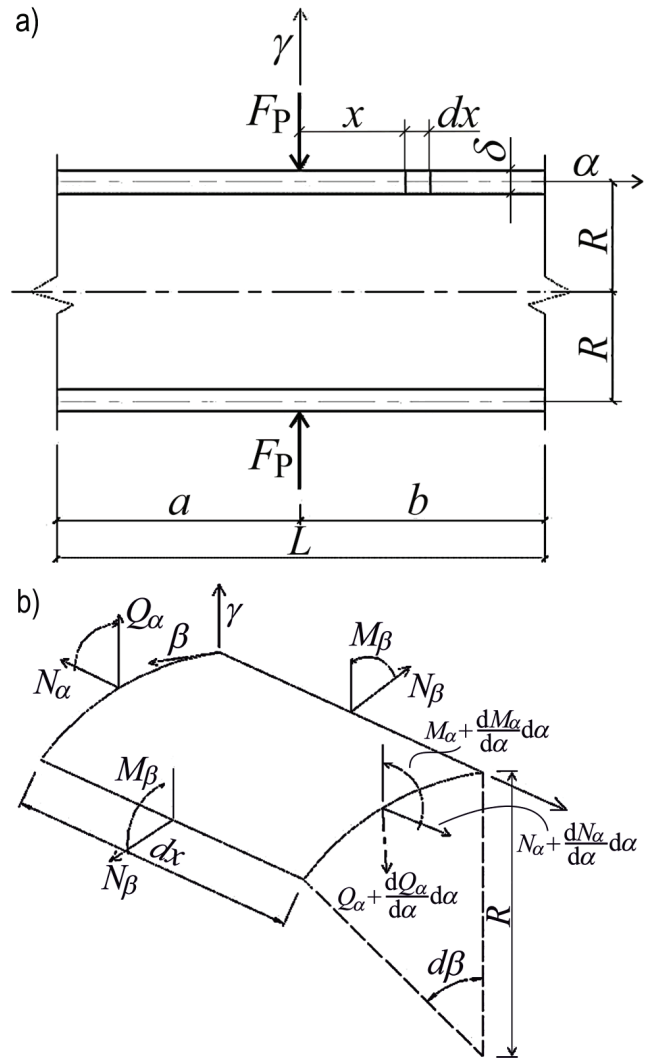

Figure 1 Geometrical parameters and stress state of prestressed concrete lining structure

Since the model of elastic mechanics established is about axisymmetric problem of cylindrical shell, according to cylindrical shell theory of elastic mechanics, the equation solving displacement and internal force is the following differential equation:

$\frac{\mathrm{d}^{4} w}{\mathrm{~d} \alpha^{4}}+\frac{E \delta}{R^{2} D} w=\frac{q_{3}}{D}$

$w$ is the normal displacement on the middle surface; $E$ is the elasticity modulus of cylinder wall material; $q_{3}$ is the normal load on the cylindric structure whose flexural rigidity is $D=\frac{E \delta^{3}}{12\left(1-\mu^{2}\right)}$ where $\mu$ is Poisson's ratio of the material.

\subsection{Model of Calculating Infinitely-Long Prestressed Concrete Liner}

Under the force of single anchor cable, prestressed concrete lining structure can be divided into infinitelylong prestressed concrete cylinder and semi-infinitelylong prestressed concrete cylinder according to the acting position of anchor cable and its distance to the top end of cylindric structure. When $a \rightarrow \infty, b \rightarrow \infty$ ( $a$ and $b$ are the acting point and the distance of equivalent radial pressure $F_{\mathrm{P}}$ to the top end), the prestressed concrete cylinder is infinitely long.
According to cylindrical shell theory of elastic mechanics, the internal force of micro-fission (Fig. 1b) meets the following conditions:

$$
\begin{aligned}
& M_{\alpha}=-\lambda^{2} D \frac{\mathrm{d}^{2} w}{\mathrm{~d} \xi^{2}}, Q_{\alpha}=-\lambda^{3} D \frac{\mathrm{d}^{3} w}{\mathrm{~d} \xi^{3}} \\
& N_{\beta}=\frac{E \delta}{R} w, M_{\beta}=\mu M_{\alpha}
\end{aligned}
$$

Here $\xi=\lambda \alpha$ and coefficient of elastic characteristics $\lambda=\sqrt[4]{\frac{E \delta}{4 R^{2} D}}=\sqrt[4]{\frac{3\left(1-\mu^{2}\right)}{R^{2} \delta^{2}}}$ which clearly shows that both the axial and circumferential internal force are directly correlated with the normal displacement of middle surface. Therefore, this can be simplified just through solving the displacement $w$ in Eq. (1).

As shown in Fig. 1a, $F_{\mathrm{P}}$ evenly distributes along the ring direction on an infinitely-long cylinder. Action point of $F_{\mathrm{P}}$ was used as the origin of coordinates to set up a cylindrical coordinate system; $q_{3}=F_{\mathrm{P}}$ was substituted into Eq. (1) to solve this equation based on the symmetric structure, thus the displacement of any cross section $\alpha$ close to anchor cable was calculated.

$$
w=-\frac{F_{\mathrm{P}}}{8 \lambda^{3} D} f_{1}(\xi)
$$

Substitute Eq. (3) into Eq. (2) and intersection angle $\theta\left(\theta=\frac{\mathrm{d} w}{\mathrm{~d} \alpha}=\lambda \frac{\mathrm{d} w}{\mathrm{~d} \xi}\right)$

$N_{\beta}=\frac{E \delta}{R} w=-\frac{F_{\mathrm{P}} E \delta}{8 \lambda^{3} D R} f_{1}(\xi)$,

$\sigma_{\beta}=\frac{N_{\beta}}{\delta}=-\frac{F_{\mathrm{P}} E}{8 \lambda^{3} D R} f_{1}(\xi)$

$M_{\alpha}=-\lambda^{2} D \frac{\mathrm{d}^{2} w}{\mathrm{~d} \xi^{2}}=-\frac{F_{\mathrm{P}}}{4 \lambda} f_{3}(\xi)$,

$Q_{\alpha}=-\lambda^{3} D \frac{\mathrm{d}^{3} w}{\mathrm{~d} \xi^{3}}=\frac{F_{\mathrm{P}}}{2} f_{4}(\xi)$,

$\theta=\frac{\mathrm{d} w}{\mathrm{~d} \alpha}=\lambda \frac{\mathrm{d} w}{\mathrm{~d} \xi}=\frac{F_{\mathrm{P}}}{4 \lambda^{2} D} f_{2}(\xi)$

$\sigma_{\beta}$ is the circumferential stress and $f_{1}(\xi)=\mathrm{e}^{-\xi}(\cos \xi+\sin \xi)$, $f_{2}(\xi)=\mathrm{e}^{-\xi} \sin \xi, f_{3}(\xi)=\mathrm{e}^{-\xi}(\cos \xi-\sin \xi), f_{4}(\xi)=\mathrm{e}^{-\xi} \cos \xi$. If $\xi=\lambda \alpha$ is large enough, these 4 special functions $f_{1}(\xi) \approx f_{4}(\xi)$ will have increasingly smaller values, which implies that under the force of single anchor cable, the normal displacement and its internal force are local [9]. When $\xi=\lambda \alpha>\pi$ the absolute value of each special function is smaller than $5 \%$ of its maximum absolute value, when $\alpha>\pi / \lambda=\pi \sqrt[4]{\frac{R^{2} \delta^{2}}{3\left(1-\mu^{2}\right)}}=2 \sqrt{R \delta} \approx 2.5 \sqrt{R \delta}$. In other words, $2 \sqrt{R \delta} \approx 2.5 \sqrt{R \delta}$ farther away from the stressed cross section, the normal displacement and internal force is too 
small to consider, so the model of calculating infinitelylong stressed concrete lining cylinder can be applied.

\subsection{Model of Calculating Semi-Infinitely-Long Prestressed Concrete Liner}

When $a \leq \pi / \lambda, b \rightarrow \infty$ or $b \leq \pi / \lambda, a \rightarrow \infty$, the prestressed concrete cylinder is semi-infinitely-long, which is shown in Fig. 2.

Since $a \leq \pi / \lambda, b \rightarrow \infty$, the influence of load on end A has to be considered with $F_{\mathrm{P}}$ on the cylinder. Make end A as the origin of coordinates, the internal force and normal displacement can be worked out through superposition according to superposition principle. The superposition is as follows:

The cross section A of this concrete cylinder can be extended into an infinitely-long cylinder and it can be assumed that the bending moment and shearing force of $F_{\mathrm{P}}$ on $\mathrm{A}$ are $M_{\mathrm{A}}$ and $F_{\mathrm{SA}}$. Since the internal force and normal displacement on A of semi-infinitely-long prestressed concrete cylinder are 0 , reverse acting force $M_{\mathrm{A}}$ and $F_{\mathrm{SA}}$ were applied on A of infinitely-long prestressed concrete cylinder to offset the internal force in order to meet the boundary conditions of null hypothesis. So the normal displacement and internal force on each cross section of semi-infinitely-long prestressed concrete cylinder can be worked out through the superposition of normal displacement and internal force on cross section of infinitely-long prestressed concrete cylinder bearing external load and force.

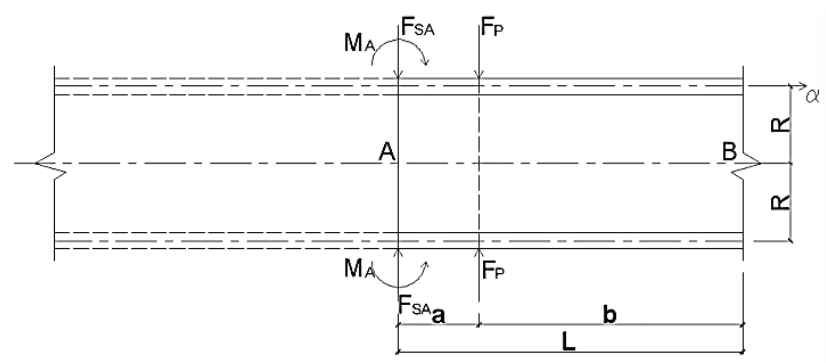

Figure 2 Theoretical model of calculating semi-infinitely-long prestressed concrete cylinder

According to the calculation of external load born by infinitely-long prestressed concrete cylinder, $M_{\mathrm{A}}$ and $F_{\mathrm{SA}}$ are

$M_{\mathrm{A}}=-\frac{F_{\mathrm{P}}}{4 \lambda} f_{3}(a \lambda), F_{\mathrm{SA}}=\frac{F_{\mathrm{P}}}{2} f_{4}(a \lambda)$

According to the above superposition, the normal displacement caused by the prestress of single anchor cable on semi-infinitely-long prestressed concrete cylindrical structure is:

$w=\frac{F_{\mathrm{P}}}{8 \lambda^{3} D}\left[\frac{f_{3}(a \lambda)}{2} f_{3}(\xi)-f_{4}(a \lambda) f_{4}(\xi)-f_{1}(|a \lambda-\xi|)\right]$

The internal force on the wall of this cylinder can be calculated through Eqs. (4) $\div(8)$.

\section{ANALYSIS ON THE SCOPE OF STRESS'S INFLUENCE ON PRESTRESSED CONCRETE LINING STRUCTURE}

With the data observed through a permanent observation apparatus in a China's un-bonded prestressed concrete lining project, the influence scope of stress of single anchor cable on the cylindrical structure was calculated theoretically.

Un-bonded prestressed concrete liner is used in Xiaolangdi Desalting Tunnel project [12]; the highest design level during operating period is $120 \mathrm{~m}$; one segment of concrete pouring runs as long as $12 \mathrm{~m}$ and the elasticity modulus of lining concrete is about $32.5 \mathrm{GPa}$; the liner was designed as thick as $0.65 \mathrm{~m}$ and its inner diameter is $6.50 \mathrm{~m}$; space between anchor cables is 0.50 $\mathrm{m}$ and the cable consists of 8 unbounded steel strands which are strong and loose and the tension of single anchor cable is designed at $1674 \mathrm{kN}$. These steel strands wind in double rings along the tunnel; the anchorage slot is arrayed in two symmetric rows along the axial direction of tunnel at the bottom of liner and the intersection angle of centre between two adjacent slots is $90^{\circ}$. One pouring segment $\mathrm{AB}$ in the observation apparatus is cut out to be used as the object of study. The layout of $A B$ segment of prestressed anchor cable is displayed in Fig. 3.

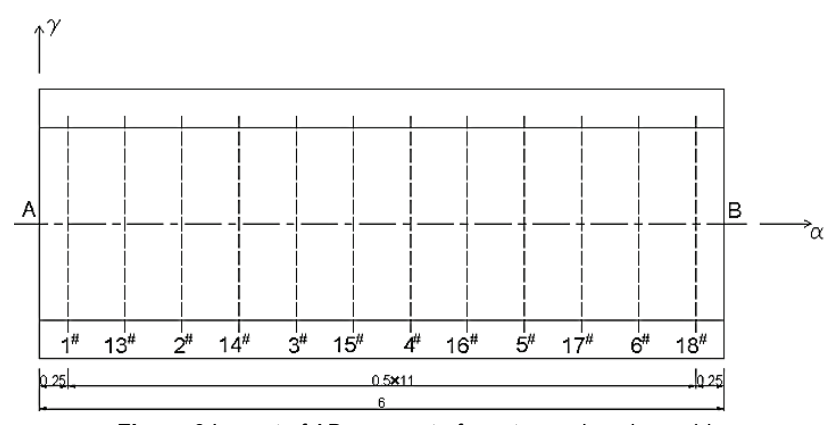

Figure 3 Layout of $A B$ segment of prestressed anchor cable

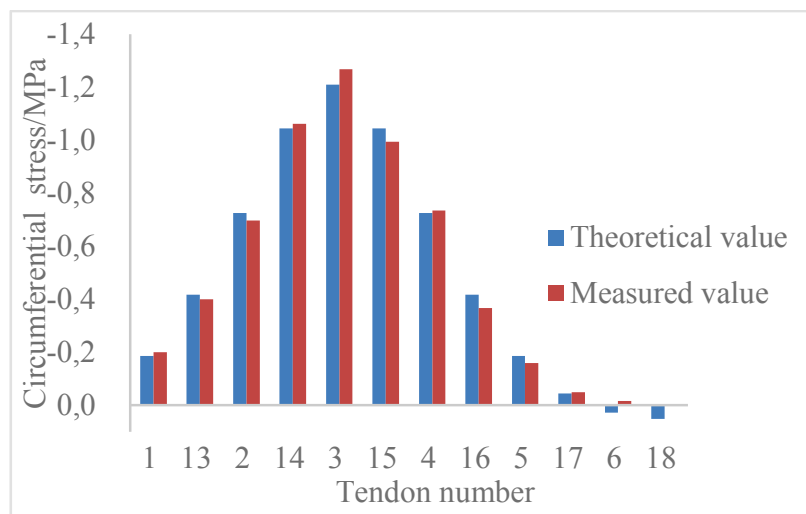

Figure 4Theoretical value and measured value of circumferential force on cross section with the stretch-draw of $3^{\#}$ anchor cables

Scope of influence of single anchor cable's stress was measured in site according to the record of stretch-draw of $3^{\#}$ anchor cables observed through the permanent observation apparatus. After theoretical analysis, there is $\alpha_{\max }=\pi \sqrt[4]{\frac{R^{2} \delta^{2}}{3\left(1-\mu^{2}\right)}}=2.41 \sqrt{R \delta}(\mu=0.2)$, which reveals that the size of influence scope is only related to the diameter of middle surface $R$ and thickness of cylinder 
wall $\delta$. Since segment $\mathrm{AB}$ is one part of the whole pouring segment, distance from $3^{\#}$ anchor cables to the two ends of pouring segment is larger than $\alpha_{\max }$, therefore, this project can be seen as an infinitely-long cylinder for calculation. With the stretch-draw of $3^{\#}$ anchor cables, the theoretical value of circumferential force can be calculated through Eq. (5) and the results are shown in Fig. 4.

It can be seen that when stretching $3^{\#}$ anchor cables, the circumferential force produced on the cross section is strongest while the circumferential force on the cross section of adjacent cables will become smaller and smaller to 0 at the location $3 \mathrm{~m}$ away from the cables. Consequently, the largest scope of influence observed through the apparatus is about $3.3 \mathrm{~m}$. Result from theoretical calculation is $3.5 \mathrm{~m}$ which is quite similar to the measured result-only $5 \%$ difference.

\section{METHOD OF DETERMINING MAXIMUM CABLE SPACING IN PRESTRESSED CONCRETE LINING STRUCTURE}

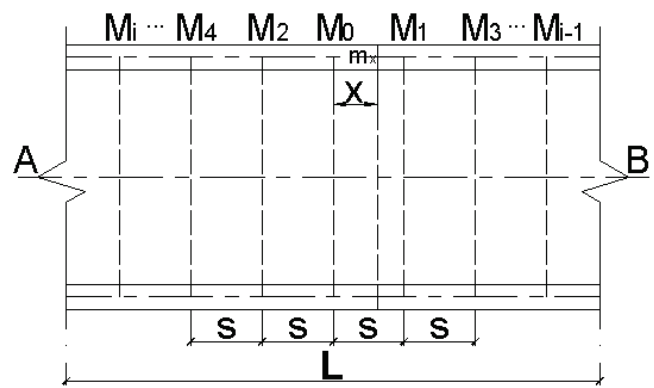

Figure $\mathbf{5}$ Model of calculating maximum cable spacing in prestressed concrete lining structure

Model of calculating infinitely-long concrete liner was used. This model in axial direction is as long as $L$; elasticity modulus of lining structure concrete is $E$; thickness and inner diameter of the liner are $\delta$ and $R$, and the tensile force of single anchor cable is designed as $F_{\mathrm{P}}$ and the spacing is $s$. The distance of some point $\mathrm{mx}$ on the middle surface between two cables $M_{0}$ and $M_{1}$ displayed in Fig. 5 is $x(x<s)$. The normal displacement, circumferential stress, bending moment and shearing force at this point are the concurrent result of all cables in the influence scope of the stress. The superposition formula at point $m_{x}$ is:

$$
\begin{aligned}
& w=\sum_{i=0}^{n} w_{i}=-\frac{F_{P}}{8 \lambda^{3} D} \sum_{i=0}^{n}\left[f_{1}\left(\xi_{2 i}\right)+f_{1}\left(\xi_{2 i+1}\right)\right] \\
& \sigma_{\beta}=\sum_{i=0}^{n} \sigma_{\beta i}=-\frac{F_{P} E}{8 \lambda^{3} D E R} \sum_{i=0}^{n}\left[f_{1}\left(\xi_{2 i}\right)+f_{1}\left(\xi_{2 i+1}\right)\right](12) \\
& \theta=\sum_{i=0}^{n} \theta_{i}=-\frac{F_{P}}{4 \lambda^{2} D} \sum_{i=0}^{n}\left[f_{1}\left(\xi_{2 i}\right)+f_{1}\left(\xi_{2 i+1}\right)\right] \\
& \xi_{2 i}=\lambda(i s+x), \quad \xi_{2 i+1}=\lambda\left[f_{1}(i+1) s-x\right](i=0,1,2,3, \ldots, n) .
\end{aligned}
$$

On the completion of stretch-draw of all cables, circumferential force on any point $m_{x}$ on the middle surface is the same, which is an ideal situation. In practical engineering, on the acting surface (that is $x=0$ ), the circumferential stress is the maximum $\sigma_{\beta \max }$ and the counterpart in the middle of two adjacent cables (that is $x=0.5 \mathrm{~s})$ is the minimum $\sigma_{\beta \min }$. To ensure an even distribution of circumferential force in axial direction inside the cylinder, the proportion of $\sigma_{\beta \min }$ to $\sigma_{\beta \max }$ must be set at allowable value according to different engineering requirements and then this value can be used to determine the maximum space between cables $s_{\max }$.

\section{CALCULATION EXAMPLE}

The water delivery tunnel of one reservoir at the second-stage of project is a pressure tunnel which is as long as $24.5 \mathrm{~km}$ and its inner diameter is $6 \mathrm{~m}$; the highest design level during operating period is $55 \mathrm{~m}$ and unbounded prestressed concrete lining structure was used. The strength grade, thickness and Poisson's ratio of lining concrete are $\mathrm{C} 40,0.5 \mathrm{~m}$ and 0.2 respectively. Epoxy coated un-bounded rebar $7 \varnothing 5$ was used in the prestressed steel strand and its maximum tensile force is $1395 \mathrm{MPa}$ and the ratio of $\sigma_{\beta \min }$ to $\sigma_{\beta \max }$ should be not more than 90 $\%$ in this project. Circumferential force between two adjacent cables can be calculated according to the method of determining maximum cable spacing and through superposition Eq. (12). The calculating is:

Theoretical calculation model (Fig. 5) was established according to conditions given and relevant parameters were calculated:

Flexural rigidity $D=\frac{E \delta^{3}}{12\left(1-\mu^{2}\right)} \approx 352647.57 \quad \mathrm{kN} \cdot \mathrm{m}$.

Coefficient of elastic characteristics

$\lambda=\sqrt[4]{\frac{E \delta}{4 R^{2} D}}=\sqrt[4]{\frac{3\left(1-\mu^{2}\right)}{R^{2} \delta^{2}}} \approx 1.022 \mathrm{~m}$.

The maximum scope of influence of stress is:

$$
\begin{aligned}
& \alpha_{\max }=\frac{\pi}{\lambda}=\pi \sqrt[4]{\frac{R^{2} \delta^{2}}{3\left(1-\mu^{2}\right)}}=2.41 \sqrt{R \delta} \approx 3.0 \mathrm{~m} \\
& \Phi=-\frac{F_{\mathrm{P}} E}{8 \lambda^{3} D E R}=-1.5
\end{aligned}
$$

1) Suppose that the spacing is $s^{\prime}=0.4 \mathrm{~m}$ and that $\sigma_{\beta \min }$ is located in the middle between $M_{0}$ and $M_{1}$ (that is $x=0.2$ m). $\sigma_{\beta \min }$ was superimposed through Eq. (12) and in this formula, there are $n=2 \frac{\alpha_{\max }}{s}=2 \frac{3}{0.4}=15$, constant number $\Phi=-\frac{F_{\mathrm{P}} E}{8 \lambda^{3} D E R}=-1.5 \mathrm{MPa}$.

2) Circumferential stress at the location in the middle between $M_{0}$ and $M_{1}$ (that is $x=0.2 \mathrm{~m}$ ) was calculated:

$$
\begin{aligned}
& M_{0}: \sigma_{\beta 0}=\Phi f_{1}\left(\xi_{0}\right)=-1.5 \mathrm{MPa}, \xi_{0}=0.2 \lambda \text {, } \\
& M_{1}: \sigma_{\beta 1}=\Phi f_{1}\left(\xi_{1}\right)=-1.5 \mathrm{MPa}, \xi_{1}=(s-0.2) \lambda \text {, } \\
& M_{2}: \sigma_{\beta 2}=\Phi f_{1}\left(\xi_{2}\right)=-1.2 \mathrm{MPa}, \xi_{2}=(s+0.2) \lambda \text {, } \\
& M_{3}: \sigma_{\beta 3}=\Phi f_{1}\left(\xi_{3}\right)=-1.2 \mathrm{MPa}, \xi_{3}=(2 s-0.2) \lambda \text {, } \\
& M_{4}: \sigma_{\beta 4}=\Phi f_{1}\left(\xi_{4}\right)=-0.8 \mathrm{MPa}, \xi_{4}=(2 s+0.2) \lambda \text {, } \\
& M_{5}: \sigma_{\beta 5}=\Phi f_{1}\left(\xi_{5}\right)=-0.8 \mathrm{MPa}, \xi_{5}=(3 s-0.2) \lambda \text {, } \\
& M_{14}: \sigma_{\beta 14}=\Phi f_{1}\left(\xi_{14}\right)=-0.1 \mathrm{MPa}, \xi_{14}=(7 s+0.2) \lambda \text {, } \\
& M_{15}: \sigma_{\beta 15}=\Phi f_{1}\left(\xi_{15}\right)=-0.1 \mathrm{MPa}, \xi_{15}=(8 s-0.2) \lambda \text {, }
\end{aligned}
$$


$\sigma_{\beta \min }=\sum_{i=0}^{15} \sigma_{\beta i}=-7.8 \mathrm{MPa}$

3) The maximum circumferential stress was calculated through the superposition formula, which is $\sigma_{\beta \max }=-7.9$ $\mathrm{MPa}$.

4) A new round of calculation was done based on new spacing $\mathrm{s}$ and the calculating process is the same as the above one. Results are presented in Tab. 1.

Table 1 Trial calculated results of circumferential stress

\begin{tabular}{|c|c|c|c|}
\hline Cable spacing $s / \mathrm{m}$ & $\sigma_{\beta \max } / \mathrm{MPa}$ & $\sigma_{\beta \min } / \mathrm{MPa}$ & $\sigma_{\beta \min } / \sigma_{\beta \max }$ \\
\hline 0.4 & 7.9 & 7.8 & 0.987 \\
\hline 0.5 & 6.3 & 6.2 & 0.984 \\
\hline 0.6 & 5.3 & 5.2 & 0.981 \\
\hline 1.0 & 3.2 & 3.1 & 0.969 \\
\hline 1.5 & 2.2 & 2.1 & 0.955 \\
\hline 2.0 & 1.7 & 1.4 & 0.824 \\
\hline 2.5 & 1.5 & 1.1 & 0.733 \\
\hline
\end{tabular}

It can be clearly seen that when $s$ is certain, values of $\sigma_{\beta \max }$ and $\sigma_{\beta \min }$ will decrease gradually with the increase of spacing. Trail calculation reveals that when $s=1.5 \mathrm{~m}$, $\sigma_{\beta \min } / \sigma_{\beta \max }$ will be $0.955>0.9$. So it can be made clear that $s_{\max }=1.5 \mathrm{~m}$ and that when $s \leq s_{\max }$, the circumferential stress caused by the stretch-draw of cable in the prestressed liner concrete distributes evenly in the axial direction. In constructed projects, since $s<s_{\max }$, it is easy for circumferential stress to distribute evenly in the axial direction, thus the even distribution is not a decisive factor for cable spacing.

\section{CONCLUSION}

(1) Theoretical analysis reveals that the formula of maximum scope of influence is $\alpha_{\max }=\pi / \lambda$, and the theoretical result coincides with the measured result.

(2) The maximum cable spacing can be determined through Eqs. (11) $\div(13)$, which is also applicable in practical projects.

(3) The even distribution of circumferential stress caused by the stretch-draw of anchor cable is not a decisive factor for determining the cable spacing.

\section{Acknowledgements}

This work was financially supported by National Natural Science Foundation of China (50979068).

\section{REFERENCES}

[1] Kang, J. F., Liang, Y. H. \& Zhang, Q. C. (2006). 3-D Finite Element Analysis of Post-Prestresed Concrete Lining. Journal of Tianjin University, 39(8), 968-972.

[2] Ministry of Water Resources of the People's Republic of China. (2004). DL/T5195-2004 Specification For Design of Hydraulic Tunnel. Beijing: China Electric Power Press.

[3] Jingfu, K. \& Yuming, H. (2005). Techniques and Performance of Post-Prestressed Tunnel Liner. Practice Periodical on Structural Design and Construction, 10(2), 102-108.

https://doi.org/10.1061/(ASCE)1084-0680(2005)10:2(102)
[4] Goel, R. K., Swarup, Anil \& Sheorey, P. R. (2007). Bolt length requirement in underground openings. Int. J. Rock Mech. \& Min. Sci., 44, 802-811. https://doi.org/10.1016/j.jirmms.2006.12.001

[5] Stacey, T. R. \& Yu, X. (2004). Investigations into mechanisms of rock support provided by sprayed liners. Ground Support in Mining and Underground Construction. Villaescusa and Potvin (eds.). London : Taylor and Francis Group.

[6] Gabriel F. (1994). Behaviour of Pressure Tunnels and Guidelines for Liner Design. Journal of Geotechnical Engineering, 120(10), 1768-1791. https://doi.org/10.1061/(ASCE)0733-9410(1994)120:10(1768)

[7] Valdeolivas, J. L. G. \& Mosquera, J. C. (2016).ThreeDimensional FEM Parametric Analysis of Stiffened Steel Liners in Hydroelectric Pressure Tunnels. Journal of Pipeline Systems Engineering and Practice, 7(1), 04015019 https://doi.org/10.1061/(ASCE)PS.1949-1204.0000218

[8] Yin, J. \& Cao, S. R. (2014). Impact and Selection of Anchor Interval on Strength of Prestressed Lining Structure. Water Resources and Power, 32(6), 104-108.

[9] Xu, Z. L. (1982). Mechanics of Elasticity. Beijing: Higher Education Press.

[10] Li, X. K., Zhao, S. B. \& Zhao, G. F. (2004). Design Methods of Prestressed Concrete Penstock. Engineering Mechanics, 21(6), 124-130+155.

[11] Lin, X. S., Shen, F. S. \& Zhang, Y. (1999). Study and Practice on the Post-Tensioned Sediment Tunnel Liner of Xiao Langdi Project. Zhengzhou : Huanghe Water Press.

[12] Yu, X. R., Kang, J. F. \& Li, X. P. (2000). Construction Technique of Concrete Tunnel Liner Post-Tensioned with Double-Looped Unbonded Tendons. Beijing : China Water Conservancy and Hydropower Press.

[13] Hetényi, A. (1950). A General Solution for the Bending of Beams on an Elastic Foundation of Arbitrary Continuity. $J$ Appl Phys, 21(55), 42-44. https://doi.org/10.1063/1.1699420

[14] Timoshenko, S. \& Woinowsky-Krieger, S. (1977). Theory of Plates and Shells. Beijing: Science Press.

\section{Contact information}

\section{Rui JING, PhD}

(Corresponding author)

School of Civil Engineering, Tianjin University

No. 92, Weijin Road, Nankai District, Tianjin, China, 300072

E-mail: michael_jing2016@yahoo.com

\section{Jingfu KANG, Prof. PhD}

School of Civil Engineering, Tianjin University

No. 92, Weijin Road, Nankai District, Tianjin, China, 300072

E-mail: kangjingfu@126.com

\section{Zhen WANG, PhD student}

Department of Civil and Environmental Engineering,

Korea Advanced Institute of Science and Technology (KAIST)

E-mail: zhenw2015@yahoo.com

Zhaowei SHEN, Undergraduate for Master

Shandong Electric Power Engineering Consulting Institute

E-mail: shenzhaowei@sdepci.com

Dazhi WANG, Undergraduate for Master

School of Civil Engineering, Tianjin University

No. 92, Weijin Road, Nankai District, Tianjin, China, 300072

E-mail: 1084509285@qq.com 